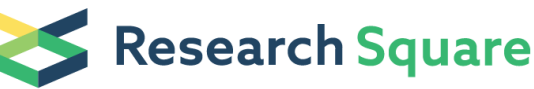 \\ Preprints are preliminary reports that have not undergone peer review. \\ They should not be considered conclusive, used to inform clinical practice, or referenced by the media as validated information.
}

\section{A Randomized Clinical Trial on the Effect of Foot Reflexology in the Fourth Stage of Labor on Uterine After Pain}

\section{Neda Sharifi}

MSc in Midwifery, Department of Midwifery, Mashhad Branch, Islamic Azad University, Mashhad, Iran. Narjes Bahri ( $\square$ nargesbahri@yahoo.com )

Assistant Professor in Reproductive Health, Social Development \&Health Promotion Research Center, Department of Midwifery, School of Medicine, Gonabad University of Medical Sciences, Gonabad, Iran.

\section{Fateme Hadizade Talasaz}

Assistant Professor in Reproductive Health, Social Development \&Health Promotion Research Center, Department of Midwifery, School of Medicine, Gonabad University of Medical Sciences, Gonabad, Iran.

\section{Hoda Azizi}

MD, PhD, Assistant Professor, Department of Chinese and Complementary Medicine, School of Pesian and Complementary Medicine, Mashhad University of Medical Sciences, Mashhad, Iran.

\section{Hosein Nezami}

Master of Biostatistics, Department of basic science, faculty of medicine, Social Development \&amp; Health Promotion Research Center, Gonabad University of Medical Sciences, Gonabad, Iran.

\section{Research Article}

Keywords: Reflexology, after-pain, complementary medicine

Posted Date: December 21st, 2020

DOl: https://doi.org/10.21203/rs.3.rs-116158/v1

License: (c) (i) This work is licensed under a Creative Commons Attribution 4.0 International License. Read Full License 


\section{Abstract}

Objective: Uterine after-pains are among the most prevalent complaints after natural vaginal delivery. Non-pharmacological treatment modalities to relieve after-pains are an important care priority. This study aimed to investigate the effect of foot reflexology in the fourth stage of labor on after-pains in multiparous women.

Methods: This single-blind randomized clinical trial recruited 80 eligible pregnant women presenting to Allameh Bohlool Hospital in Gonabad and 17-Shahrivar Hospital in Mashhad in 2019. In the first and second hours postpartum, the reflexology group received 10 minutes of general massage and specific reflexology massage on each foot in uterine, pituitary, and solar plexus points. The control group received 10 minutes of general massage, and then rotational massage on a neutral point on the lateral side of the heel. The pain was measured every hour up to 4 hours postpartum using a visual analog scale. The collected data were analyzed in SPSS-16 software at a significance level of $P<0.05$.

Results: The median of after-pain score in the first hour postpartum was not statistically significantly different between the two groups $(P=0.05)$, but it was lower in the reflexology group at the second $(P=0.04)$, third $(P<0.001)$, and fourth hours postpartum $(P<0.001)$. The mean total after-pain score of the four hours postpartum was significantly lower in the reflexology group $(P<0.001)$.

Conclusions: The results revealed that foot reflexology in the fourth stage of labor has positive effects on the relief of uterine after-pain. It is therefore recommended that reflexology be used to reduce postpartum uterine after-pain.

\section{Introduction}

Pregnancy, labor, and puerperium are associated with many changes in women's body and may affect their health (1). Uterine after-pains are reportedly the most prevalent postpartum pain (77\%) in multiparous women (2). Uterine after-pains usually last 2 to 4 days postpartum and resolve spontaneously. Factors affecting pain intensity include multiparity, uterine overdistention (multiple pregnancies, large fetus, polyhydramnios), breastfeeding, prolonged labor stages, analgesia during labor, maternal physical and mental disorders, history of dysmenorrhea, maternal weight, and cultural factors such as race, education and religion (3-9). The severity of after-pains increases in multiparous women due to increased sensitivity of the central nervous system and decreased uterine muscle tone following multiple deliveries or uterine overextension, while nulliparous women experience little or no after-pain because their uterus has high muscle tone and contracts well $(3,4,10)$.

After-pains can lead to maternal and neonatal complications including anxiety, sleep disturbances, emotional disorders, depression, inability to care for the baby and continue breastfeeding, delays in early breastfeeding, and reduced maternal ability to perform daily chores. James et al. (2008) reported pain in the early hours postpartum as one of the most important factors in chronic postpartum pain and depression (11). Furthermore, pain and stress increase adrenaline release and decrease oxytocin release, 
which lead to the cessation of the oxytocin reflex and disrupt breast milk production in addition to the discomfort they incur to the mother (12).

The most common method used to relieve after-pains is oral analgesics such as acetaminophen and ibuprofen (13). However, some analgesics (e.g., mefenamic acid and ibuprofen) sometimes cause side effects such as nausea, vomiting, diarrhea, abdominal pain, bleeding, gastrointestinal obstruction or perforation, dizziness, drowsiness, seizures, acute renal failure, and interstitial nephritis $(14,15)$. In the United States, the serious side effects of nonsteroidal anti-inflammatory drugs (NSAIDs) have resulted in 100,000 hospitalizations and more than 16,500 deaths (15). Medication side effects have drawn attentions to non-pharmacological pain management modalities, including reflexology. A clinical trial reported a reduction in after-pains after elective cesarean section using foot reflexology (16). A systematic review study also showed that reflexology during different stages of labor reduces labor pain severity (17).

Although numerous studies have reported the effect of reflexology on labor pain and post-cesarean section pain, our extensive review of the literature up to the time of writing this paper yielded no study regarding the effects of reflexology on other stages of labor, including the fourth stage and pain after natural delivery. Since reflexology is a non-invasive modality that can be used along conventional therapies to reduce after-pains as well as the side effects of chemical medications, this study aimed to investigate the effect of foot reflexology in the fourth stage of labor on after-pains.

\section{Methods}

\section{Trial design}

The protocol of this randomized clinical trial was approved by the Ethics Committee of Gonabad University of Medical Sciences (IR.GMU.REC.1397.079). This study was also registered at the Iranian Registry of Clinical Trials on 04/02/2019 (IRCT20181214041962N1). The report of this clinical trial is based on the CONSORT 2010 checklist(18).

\section{Participants and setting}

This study enrolled pregnant women referring to Allameh Bohlool Gonabadi Hospital in Gonabad and 17 Shahrivar Hospital in Mashhad, Iran from February 2019 to July 2019. The most important inclusion criteria were no history of postpartum hemorrhage, gravida 2 or 3, gestational age of 37-42 weeks, no high-risk pregnancy, and no uterine overdistention. The exclusion criteria were unwillingness of the mother to continue participation, manual removal of the placenta and membranes, the need for additional treatments to control bleeding, neonatal birth weight over $4 \mathrm{~kg}$, postpartum bleeding less than $500 \mathrm{cc}$. The inclusion and exclusion criteria are fully described in the paper that reported its protocol (19).

\section{Sample size and randomization}


Yousefi et al. (2011) study was considered in order to determine the sample size required for comparing the two groups in $\mathrm{G}^{*}$ Power version 3.1.9.2 software (16). The t-tests were used for this purpose. In this study, the effect size $d$ was 0.712 , type I error was a maximum of $5 \%$, and test power was $80 \%$, which determined the sample size of 64 patients (32 patients in each group). Considering $20 \%$ sample loss, the final sample size was at least 40 individuals per group.

Convenience sampling was used to recruit the subjects who were then allocated to the reflexology or control group using permuted blocks of 4 . The randomization are described in detail in the protocol article of this study(19).

\section{Study instruments}

Data were collected using several questionnaires including demographic and obstetric questionnaire; labor checklist and visual analog scale (VAS).

The instruments are described in detail in the protocol article of this study (19). Items on the demographic and obstetric questionnaire included the parturient and her husband's age, their education, weight, and obstetric history i.e. gravidity, parity, postpartum bleeding, dysmenorrhea, gestational age, bleeding in pregnancy, the position of the placenta.

The checklist for labor stages included items such as vital signs during labor stages, medications used to relieve labor pain or accelerate labor, duration of labor stages, neonatal weight, placental weight, analgesics used in the fourth stage of labor.

VAS is a standard tool whose validity has been approved in previous studies including Gallagher (2002) (20). The reliability of VAS has been confirmed in various studies, including Khalilian Muvahhed et al. (2012) by the equivalent forms reliability with $r=0.91$ (21).

\section{Interventions and outcomes}

Before sampling, the researcher's skills in performing foot reflexology techniques as per the standard protocol was approved by a traditional Chinese medicine and acupuncture specialist after passing several training sessions. At the beginning of the study, demographic and obstetric questionnaires were completed by asking the mother and checking her hospital record. The researcher was present at the mother's bed during the second stage and monitored all the second and third stages of labor and recorded the necessary information in the labor checklist form. The participants were blinded to their group (single-blind design). After the third stage of labor, the researcher started the intervention in the experimental group. Room conditions, researcher's conditions, and the parturient's conditions were considered before the intervention and during reflexology. The reflexology group received four minutes of general massage on each foot and then two minutes of specific reflexology on each point of the uterus, 
pituitary, and solar plexuses in the form of rotational pressures. This was repeated in the second hour postpartum.

The control group received general massage on each foot for four minutes and then rotational pressures on a neutral point on the lateral side of the heel (placebo point) for 6 minutes. This was repeated in the second hour postpartum. VAS was marked by the mother at each time point of 1,2,3, and 4 hours postpartum in both groups to check after-pain intensity. The postpartum care was provided for both groups according to the national protocol.

\section{Statistical analysis}

The collected data were entered into the SPSS-16 software. Shapiro-Wilk test was used to check the normal distribution of quantitative variables. Mean scores of quantitative variables were compared using Mann-Whitney test, Friedman's test and Wilcoxon's test with Bonferroni correction and covariance. Significance level was set as 0.05 .

\section{Results}

In this study, 138 individuals were evaluated for eligibility and 100 individuals were included in the study and allocated into two groups (reflexology and control). During the study, 20 individuals were excluded due to receiving misoprostol, extra oxytocin, and lack of cooperation. Finally, 80 individuals (40 in the reflexology group, and 40 in the control group) were evaluated (Figure 1).

There was no statistically significant difference between the two groups in terms of demographics characters such as maternal age, husband's age, maternal education, husband's education, maternal occupation, husband's occupation, maternal weight, and history of dysmenorrhea (Table 1).

The mean scores of after-pains were higher in the reflexology group than that in the control group in the first hour postpartum, but the difference was not statistically significant ( $P=0.050)$ (Table 2$)$. The mean scores of after-pains were significantly lower in the reflexology group at time points of 2 hours $(P=0.042)$, 3 hours $(P<0.001)$, and four hours postpartum $(P<0.001)$. Furthermore, total mean score of after-pains throughout the four hours postpartum was significantly lower in the reflexology group $(P<0.001)$ (Table 2).

According to the Friedman's test, the median score of after-pains did not significantly change from the first to the fourth hour postpartum, however, the median score of after-pain in the reflexology group decreased from the first to the fourth hour postpartum $(P<0.001)$ (Figure 2$)$.

The mean dosage of mefenamic acid in the third hour postpartum was more in the reflexology group than that in the control group $(18.66 \pm 00.00 \mathrm{mg}$ vs. $11.0 \pm 30.88 \mathrm{mg})$, while the mean dosage of mefenamic acid in the fourth hour postpartum was less in the reflexology group than that in the control 
group $(6.00 \pm 39.00 \mathrm{mg}$ vs. $16.90 \pm 1.27 \mathrm{mg})$. Mann-Whitney test results indicated that the mean dosage of mefenamic acid in the third and fourth hours postpartum was significantly different between the two groups $(P<0.05)$ (Table 3$)$.

Since the two groups did not match in terms of mefenamic acid dosage in the third and fourth hours, ANOVA was used to control the effect of mefenamic acid as a confounding factor. ANOVA revealed that mefenamic acid did not have a significant effect at time points of 3 hours $(P=0.596)$ and four hours $(P=0.408)$ postpartum, and that once the effect of mefenamic acid was controlled, there was a statistically significant difference between reflexology and control groups in severity of after-pains during the fourth hour postpartum $(P<0.001)$ (Table 4).

\section{Discussion}

After-pains felt in the lower abdomen or lower back are one of the most prevalent complaints after labor (22). NSAIDs are the most common treatment for after-pains (23). Complementary medicine used for the treatment of after-pains include a variety of anti-inflammatory and pain-relieving herbs such as dill and Nigella sativa or acupressure and acupuncture that affect the body's energy channels. Since no study was found on the effect of foot reflexology in the fourth stage of labor on after-pains in our review of the literature, the results of the present study are compared and discussed with the findings of the most relevant articles on the effect of complementary medicine such as acupressure or acupuncture on afterpains as well as the use of foot reflexology to treat pain. Jenabi et al. (2011) reported the positive effect of foot reflexology on relieving pain in the first stage of labor (24). This finding is consistent with the present study findings. They used reflexology massage on uterine points (medial side of the foot, the middle point of the heel and medial ankle) to relieve pain, which is one of the points used in the present study. The similarity of the results of the two studies can be attributed to the fact that the mechanisms of pain is somewhat similar in labor and after-pain. Ghasemali et al. (201 4) and Mathew et al. (2016) also examined the effects of foot reflexology on pain in the first stage of labor $(25,26)$. Their results showed that foot reflexology reduced labor pain, which is in line with the findings of the present study. They used the same tools and interventions as those in the present study, which can justify the consistency of the results. Mokhtari et al. (2010) and Abbaspoor (2014) reported a reduction in pain after cesarean section using foot reflexology $(27,28)$. Their results are consistent with the present study. Although part of the pain after cesarean section is related to the incision site, the general nature of the pain after cesarean section and after-pains is similar and due to uterine contractions. Therefore, the pain relief mechanism in their study appears to be similar to the present study and is based on the foot reflexology massage, which stimulates and activates the neural pathways and subtle energy pathways associated with the sole and can reduce pain intensity in patients. During the massage, blood circulation is improved and the transmission of pain signals through the sensory nerves is inhibited, and pain is finally relieved by the release of endorphins and enkephalins (29). Soltani et al. (2017) used acupressure but it did not reduce after-pains in the first and second hours postpartum (30). Their failure to reduce after-pains in the first hour postpartum is consistent with the present study, but that of the second hour postpartum is not. This difference can be attributed to the place and time of the intervention. In Soltani's study, the main 
acupressure group received acupressure first on the CV6 point ( 1.5 cun below the umbilicus) vertically and continuously for one minute after delivery at a pressure of $4-5 \mathrm{~kg} / \mathrm{cm} 2$ and then on the CV4 point (3 cun below the umbilicus). Meanwhile, in the present study, the reflexology group first received 6 minutes of general massage on each foot at the beginning of the fourth stage of labor, and then specific reflexology for at least 1 minute on each point of uterus, pituitary, and solar plexus, which were repeated in the second hour postpartum. Furthermore, other differences between the two studies include the time of measuring pain intensity and participants' gravidity, that is, in Soltani's study the pain intensity of women with gravida 5 or lower was measured at one and two hours postpartum, but in the present study, the severity of pain in women with gravida 2 and 3 was measured up to four hours postpartum. The limitations of the present study failure to confirm the accuracy of participant's statements due to ethical constraints and the influence of individual and genetic characteristics on the pain tolerance.

\section{Conclusions}

The results of the present study showed that foot reflexology in the fourth stage of labor has positive effects on and can relieve after-pains. Therefore, it is recommended that foot reflexology be used in clinic for postpartum care as a modality without side effects to improve midwifery services.

\section{Abbreviations}

RCT: Randomised controlled trial; VAS: Visual analogue scale; IRCT: Iranian Registry of Controlled Trial.

\section{Declarations}

\section{Acknowledgements}

The present study was extracted from an approved master's thesis in midwifery at Gonabad University of Medical Sciences, funded by the Research and Technology Deputy (Grant number: A1016741). We sincerely thank the Research Deputy of Gonabad University of Medical Sciences for their financial support.

\section{Authors' contributions}

NSh, NB, FH, HA and HN contributed to the conception or design of the research protocol.

NSh and NB formulated and revised the research protocol critically for important intellectual content. HN reviewed the statistical methods and sample size calculations. NSh, NB, FH, HA and HN reviewed the drafts and approved the final version of the article.

\section{Funding}

This research was funded by the research council of Gonabad University of Medical Sciences (grant number: A1016741). The funding body did not have any role in the study design, data analysis, 
manuscript preparation and submission.

\section{Availability of data and materials}

The datasets used and/or analyzed during the current study are available from the corresponding author on reasonable request.

\section{Ethics approval and consent to participate}

All participants provided written informed consent for participating the study. The trial has ethical was approved by Regional Committee on Ethics in Medical Research at Gonabad University of Medical Sciences on 03/12/2018(Approval ID: IR.GMU.REC.1397.079). All the experimental protocols for involving humans was in accordance to guidelines of national/institutional in the manuscript. This study was also registered at the Iranian Registry of Clinical Trials on 04/02/2019 (IRCT20181214041962N1). All participants' information was kept in a personal file and collected in a locked office with limited access.

\section{Consent for publication}

Not applicable

\section{Competing interests}

The authors declare that they have no competing interests.

\section{References}

1. Vincenzo Z, Matteo P, Valeria M, Lara G, Francesca V, Lorenzo S, et al. Pain and stress after vaginal delivery: characteristics at hospital discharge and associations with parity. Journal of Obstetrics and Gynaecology. 2019:1-5.

2. Chananeh M, Janati Ataei P, Dolatian M, Mojab F, Nasiri M. Effects of the Combination of Nigella Sativa and Mefenamic Acid and Mefenamic Acid Alone on the Severity of Postpartum Pain in Multiparous Women: A Double-Blind Clinical Trial. The Iranian Journal of Obstetrics, Gynecology and Infertility. 2018;21(4):62-71.

3. Williams JW, Cunningham FG, Leveno KJ, Bloom SL, Spong CY, Dashe JS. Williams obstetrics. 15, editor. IRAN: Golban; 2018.

4. Holdcroft A, Snidvongs S, Cason A, Doré CJ, Berkley KJ. Pain and uterine contractions during breast feeding in the immediate post-partum period increase with parity. Pain. 2003;104(3):589-96.

5. Yerby M. Pain in childbearing: key issues in management: Elsevier Health Sciences; 2000.

6. Taffazoli M, Khadem Ahmadabadi M. Assessment of factors affecting afterpain in multiparous women delivered in Mashhad 17-Shahrivar Hospital, Mashhad, Iran. Journal of Midwifery and Reproductive Health. 2014;2(1):60-5. 
7. Jangsten E, Bergh I, Mattsson LÅ, Hellström AL, Berg M. Afterpains: a comparison between active and expectant management of the third stage of labor. Birth. 2011;38(4):294-301.

8. Russell R, Reynolds F. Back pain, pregnancy, and childbirth. BMJ: British Medical Journal. 1997;314(7087):1062.

9. Jangsten E, Strand R, de Freitas EdGG, Hellström A-L, Johansson A, Bergström S. Women's Perceptions of Pain and Discomfort after Childbirth in Angola. African Journal of Reproductive Health. 2005;9(3).

10. Betts D. Postnatal acupuncture. Journal of Chinese medicine. 2005;77:5-1542.

11. Eisenach JC, Pan PH, Smiley R, Lavand'homme P, Landau R, Houle TT. Severity of acute pain after childbirth, but not type of delivery, predicts persistent pain and postpartum depression. Pain. 2008;140(1):87-94.

12. Lewallen LP, Dick MJ, Wall Y, Zickefoose KT, Hannah SH, Flowers J, et al. Toward a clinically useful method of predicting early breast-feeding attrition. Applied Nursing Research. 2006;19(3):144-8.

13. Batten $M$, Stevenson $E$, Zimmermann $D$, Isaacs $C$. Implementation of a hydrotherapy protocol to improve postpartum pain management. Journal of midwifery \& women's health. 2017;62(2):210-4.

14. Shadipour M, Simbar M, Salamzadeh J, Nasire N. A comparative study on the effects of Menstrogol and Mefenamic acid on postpartum after-pain. Iranian South Medical Journal. 2014;16(6):401-9.

15. Ebrahim Zadeh MA, Gholami K, Gharanjik U, Javadian P, S.M. EVALUATION OF DRUG INTERACTIONS OF NON-STEROIDAL ANTI-INFLAMMATORY DRUGS(NSAIDS) IN SARI INSURED PRESCRIPTIONS DURING 1999-2001. Razi Journal of Medical Sciences. 2003;10(36):489-95.

16. Razmjoo N, Yousefi F, Esmaeeli H, Azizi H, Lotfalizadeh M. Effect of foot reflexology on pain and anxiety in women following elective cesarean section. The Iranian Journal of Obstetrics, Gynecology and Infertility. 2012;15(1):8-16.

17. Sharifi N, Bahri N. The Effect of Foot Reflexology on the Severity of Labor Pain: a Systematic Review Study. The Iranian Journal of Obstetrics, Gynecology and Infertility. 2019;22(9):85-93.

18. Schulz KF, Altman DG, Moher D, the CG. CONSORT 2010 Statement: updated guidelines for reporting parallel group randomised trials. BMC Medicine. 2010;8(1):18.

19. Sharifi N, Bahri N, Talasaz FH, Azizi H, Nezami H. The Effect of Foot Reflexology in the Fourth Stage of Labor on Postpartum Hemorrhage and After Pain: A Randomized Controlled Trial Protocol. Advances in Integrative Medicine. 2020.

20. Gallagher EJ, Bijur PE, Latimer C, Silver W. Reliability and validity of a visual analog scale for acute abdominal pain in the ED. The American journal of emergency medicine. 2002;20(4):287-90.

21. Azhari S, Khalilian Muvahhed H, Tara F, Esmaeli H. Comparison the effect of sitting and kneeling positions during the second stage of labor on pain and duration of second stage of labor in nulliparous women. The Iranian Journal of Obstetrics, Gynecology and Infertility. 2013;15(38):7-16.

22. Eshkevari L, Trout KK, Damore J. Management of postpartum pain. Journal of midwifery \& women's health. 2013;58(6):622-31. 
23. Pourmaleky S, Najar S, Montazery S, Haghighizadeh MH. Comparison between the effects of Zintoma (Ginger) and Mefenamic acid on after pain during postpartum in multiparous women. The Iranian Journal of Obstetrics, Gynecology and Infertility. 2013;16(79):18-25.

24. Jenabi E, Hajiloo Mohajeran M, Torkamani M. The Effect of Reflexology on Relieving the Labor Pain. The Iranian Journal of Obstetrics, Gynecology and Infertility. 2012;14(8):34-8.

25. Mathew AM, Francis F. Effectiveness of Foot Reflexology in Reduction of Labour Pain among Mothers in Labour Admitted at PSG. International Journal of Nursing Education. 2016;8(3).

26. hajighasemali s, Amir Ali Akbari S, akbarzadeh a, heshmat r. Comparison between Effect Acupuncture on SP6 Point and Reflexology on the Severity of First-Stage Labor Pain. Pars of Jahrom University of Medical Sciences. 2014;12(4):17-22.

27. MOKHTARI NJ, SIRATI NM, SADEGHI SM, GHANBARI Z, BABATABAR DH, MAHMOUDI H. Comparison of impact of foot reflexology massage and Bensone relaxation on severity of pain after cesarean section: a randomized trial. 2010.

28. Abbaspoor Z, Akbari M, Najar S. Effect of foot and hand massage in post-cesarean section pain control: a randomized control trial. Pain Management Nursing. 2014;15(1):132-6.

29. Watson S. The effects of massage: an holistic approach to care. Nursing standard (Royal College of Nursing (Great Britain): 1987). 1997;11(47):45-7.

30. Soltani M, Azhari S, Khorsand Vakilzadeh A, Tara F, Mazloum SR. The effect of acupressure on uterine tone and pain after delivery. The Iranian Journal of Obstetrics, Gynecology and Infertility. 2017;20(9):91-100.

\section{Tables}

Table1: Baseline sociodemographic characteristics of two groups 


\begin{tabular}{|llll|}
\hline $\mathbf{p}$ & $\begin{array}{l}\text { control } \\
\text { group }\end{array}$ & $\begin{array}{l}\text { Intervention } \\
\text { Group }\end{array}$ & Variable \\
\hline $0.653^{*}$ & 29.103 .62 & 29.184 .93 & Women age(mean) \\
\hline $0.395^{\star}$ & 33.054 .11 & 33.674 .31 & Men age (mean \\
\hline $0.098^{*}$ & 9.583 .52 & 10.823 .35 & Women education (mean \\
\hline $0.595^{\star}$ & 10.253 .71 & 9.703 .79 & Men education(mean) \\
\hline $0.210^{+}$ & & & Women job, count (\%) \\
& $39(97.50 \%)$ & $39(97.50 \%)$ & Housewife \\
\hline $0.714^{+}$ & $1(2.50 \%)$ & $1(2.50 \%)$ & Employee \\
\hline & $16(40 \%)$ & $17(42.5 \%)$ & Manual worker \\
\hline $0.201^{*}$ & 75.151 .02 & 71.307 .81 & Women weight (mean \\
\hline 0.823 & $51.25 \%$ & $52.5 \%$ & Dysmenorrhea \\
\hline
\end{tabular}

* Mann-Whitney Test was used

+ Chi-square test was used

Table2: Comparison of postpartum pain in the first to fourth hour after delivery in two groups

\begin{tabular}{|llll|}
\hline${ }^{*} \mathbf{p}$ & $\begin{array}{l}\text { control } \\
\text { group, mean } \pm \text { SD }\end{array}$ & $\begin{array}{l}\text { Intervention } \\
\text { group, mean } \pm \text { SD }\end{array}$ & \\
\hline 0.050 & 2.051 .70 & 2.821 .82 & Variable \\
\hline 0.042 & 2.551 .10 & 2.152 .00 & Postpartum pain in the first hour \\
\hline$<0.001$ & 3.001 .06 & 1.381 .67 & Postpartum pain in the second hours \\
\hline$<0.001$ & 2.581 .35 & 0.651 .25 & Postpartum pain in the Fourth hours \\
\hline$<0.001$ & 10.173 .24 & 7.005 .38 & Postpartum pain in the first 4 hours \\
\hline
\end{tabular}


* Mann-Whitney test was used

Table 3: Comparison of Mefenamic acid used in the first to fourth hour after delivery in two groups

\begin{tabular}{|llll|}
\hline${ }^{*} \mathbf{P}$ & $\begin{array}{l}\text { control } \\
\text { group, mean } \pm \text { SD }\end{array}$ & $\begin{array}{c}\text { Intervention } \\
\text { group, mean } \pm \text { SD }\end{array}$ & Variable \\
\hline 0.010 & 11.300 .85 & 18.0066 .00 & Mefenamic acid used in the third hour \\
\hline 0.000 & 16.901 .27 & 6.0039 .00 & Mefenamic acid used in the Fourth hour \\
\hline
\end{tabular}

* Mann-Whitney Test

Table4: Results of analysis of covariance of postpartum pain in the third and fourth hour after delivery in the two study groups

\begin{tabular}{|lll|}
\hline${ }^{*} \mathbf{p}$ & mean \pm SD & Variable \\
\hline 0.596 & 1.320 .229 & postpartum pain in the third hours (reflexology group) \\
\cline { 2 - 3 } & 2.970 .224 & postpartum pain in the third hours (Control group) \\
\hline 0.408 & 0.651 .252 & postpartum pain in the fourth hours (reflexology group) \\
& 2.581 .357 & postpartum pain in the fourth hours (Control group) \\
\hline
\end{tabular}

*Analysis of covariance test was used

\section{Figures}




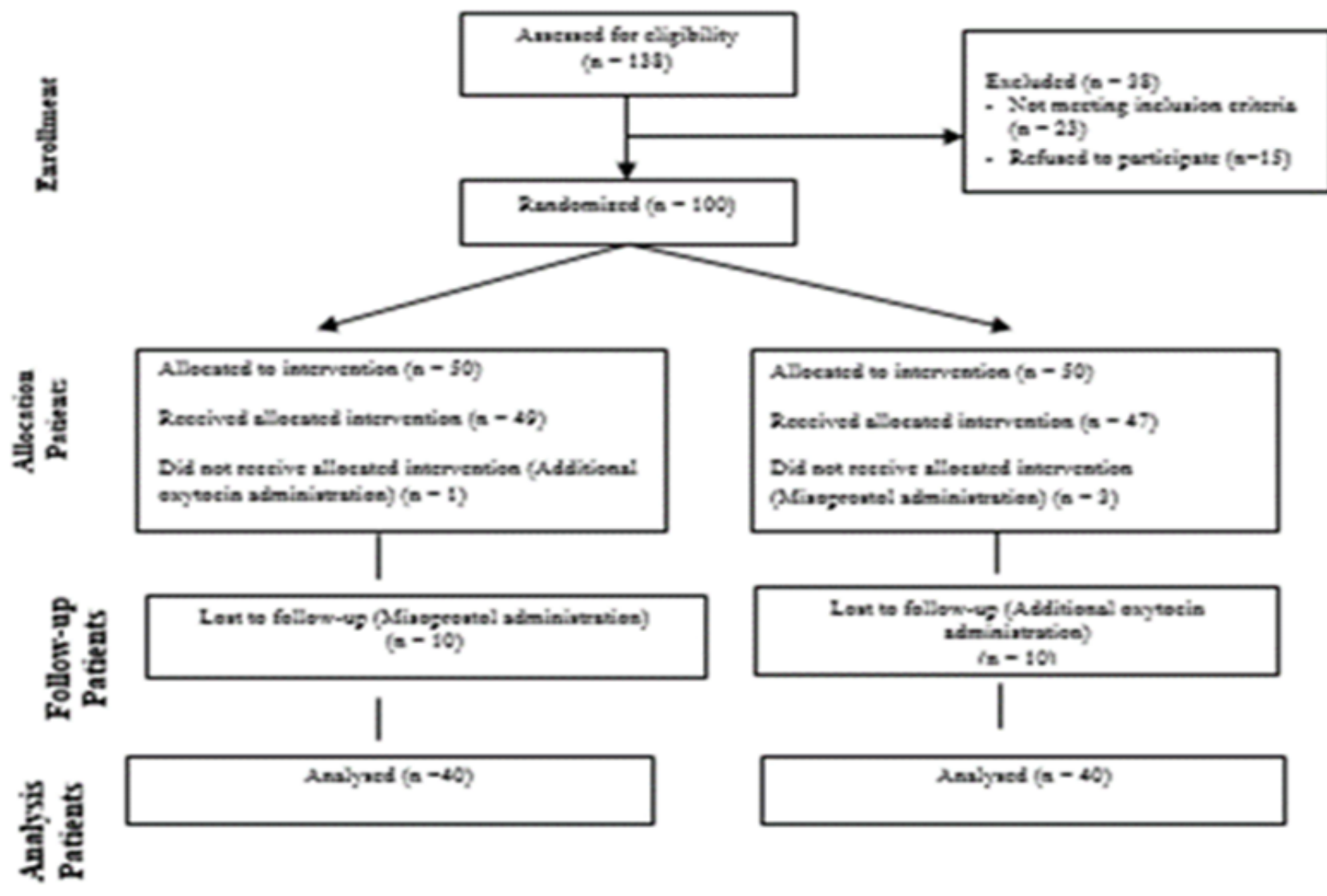

Figure 1

Representation of study flow chart

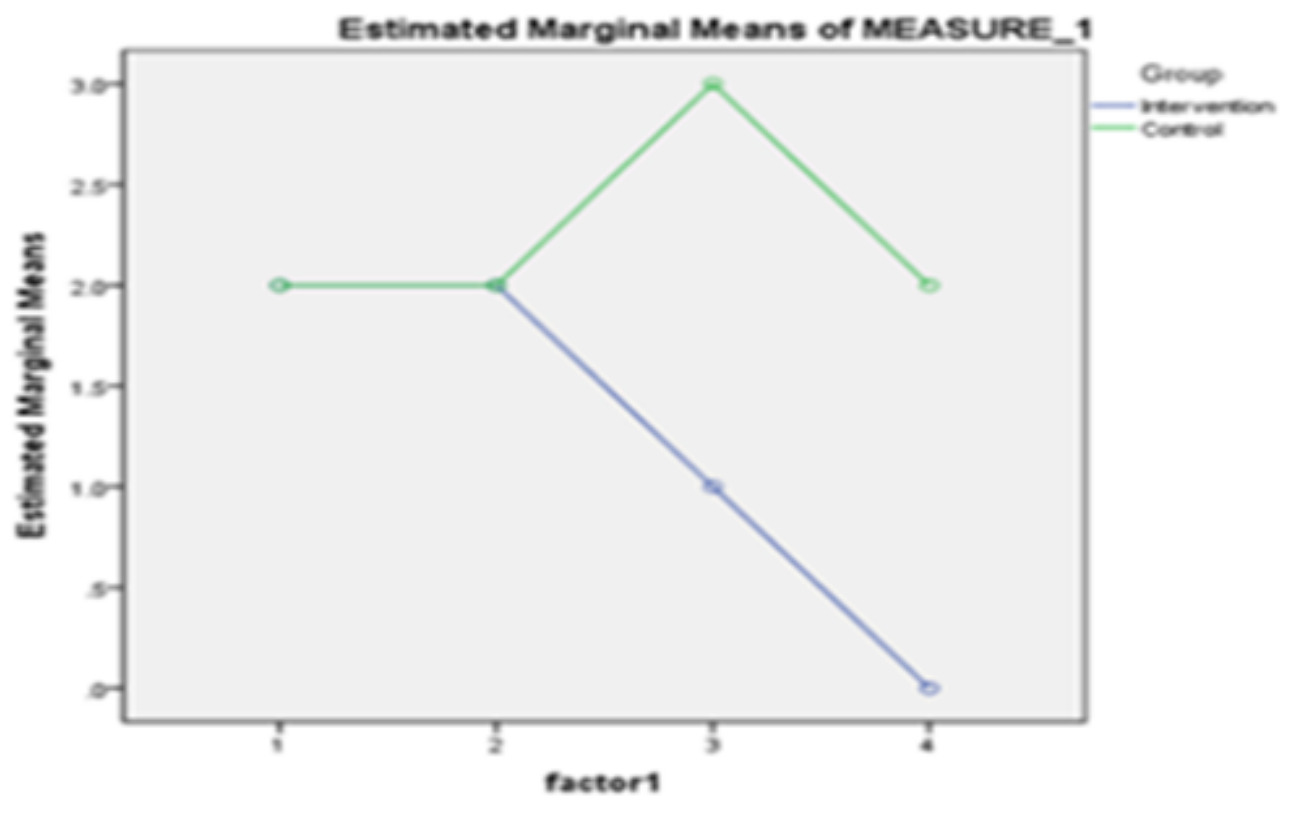

Figure 2 
Comparison of mean postpartum pain in the two study groups

\section{Supplementary Files}

This is a list of supplementary files associated with this preprint. Click to download.

- Englishversionofquestionnaire.pdf 